\title{
563 Figures
}

564

565 Figure 1. Male mating success increases with chorus attendance (number of nights monitored

566 as calling), with $49 \%$ of the variance explained by a linear regression $(n=15, \mathrm{p}<0.01)$. One

567 male was assigned a chorus attendance of zero because he started calling long after the end of 568 the reproductive period.

569

570 Figure 2. Tadpole growth rate increases with male attractiveness $a$ (number of matings

571 obtained per night of chorus attendance), with $54 \%$ of the variance explained by a linear

572 regression $(n=9, \mathrm{p}<0.01)$.

573 